\title{
An Adaptive Feedback Mechanism for Consensus Reaching Processes Based on Individuals' Credibility
}

\author{
Francisco Javier Cabrerizo \\ University of Granada \\ cabrerizo@decsai.ugr.es
}

\author{
Ignacio Javier Pérez \\ University of Cádiz \\ ignaciojavier.perez@uca.es
}

\author{
Juan Antonio Morente-Molinera \\ International University of La Rioja \\ juan.morente@unir.net
}

\author{
Sergio Alonso \\ University of Granada \\ zerjioi@ugr.es
}

\begin{abstract}
In group decision making, the decision made should be the one of highest consensus. Numerous consensus reaching processes have been developed as a part of the decision process. Within a consensus reaching process, a notable step is that of the feedback mechanism that helps the individuals arrive at an agreement by generating some recommendations on how they should modify their evaluations. Our objective is to present a new feedback mechanism based on individuals' credibility. Different from the existing approaches, it adapts the recommendations provided to the individuals depending on their credibility.
\end{abstract}

\section{Introduction}

In the setting of this study, group decision making is a kind of participatory process where more than one individual, $E=\left\{e^{1}, \ldots, e^{m}\right\}$, discusses a problem collectively, consider a collection of options, $X=$ $\left\{x_{1}, \ldots, x_{n}\right\}$, to solve it, and evaluate them [1]. To do so, two processes are carried out sequentially. The first one, the consensus reaching process $[2,3]$, is a creative and dynamic way of achieving agreement among all individuals of the group, which are committed to finding a solution supported by all, or at least a solution that everyone can live with. The second one, the selection process [4], obtains the final solution in consonance with the evaluations provided. As a result, we arrive at a rank of options from best to worst to solve the problem.

A notable step of the consensus reaching process, which is called feedback mechanism, is that of providing advice about how the individuals should modify their evaluations with the aim of increasing the consensus. At first, a moderator carried out this task [5]. However, since the moderator may introduce some subjectivity in the process, automatic tools substituted her/him:

\author{
Enrique Herrera-Viedma \\ University of Granada \\ viedma@decsai.ugr.es
}

- Some approaches incorporated a feedback mechanism whose behavior remains fixed through the whole consensus reaching process $[3,6]$. They are considered as rigid or inflexible.

- Some approaches incorporated a feedback mechanism adapting its operation to the consensus reached in each discussion round [7]. These approaches present a faster convergence towards the consensus than the rigid ones [8].

However, these approaches either do not consider the knowledge level of the individuals or only consider it in the selection process when aggregating the preferences provided by the individuals [9]. A new consensus approach was presented in [1] to improve the above approaches. It incorporated a feedback mechanism customizing the advice needed by each individual according to her/his level of importance (knowledge level). Its drawback is the way in which the importance level is assigned to the individuals as it is done subjectively at the beginning of the decision process. Therefore, it presents a similar problem that the approaches with a moderator.

The objective of this study is to present, as a part of a consensus reaching process, a new feedback mechanism based on individuals' credibility. Argumentation is a human feature that allows people to handle discussions by exchanging arguments in a competitive or cooperative fashion to reach a final agreement or just to express an individual position [10]. In such a way, any consensus reaching process should implement some argumentation mechanism in order to imitate the human being way to manage negotiation processes [11]. In addition, the key to believe or refuse arguments given by other people is usually their credibility [12]. To assess the credibility of the individuals could play a crucial role when we carry out a consensus reaching process. Therefore, we introduce a segmentation mechanism that divides the individuals into different subsets (very 
credible, credible and hardly credible). Then, if the individuals of the group do not reach a consensual agreement, this feedback mechanism sends them some recommendations to get their opinions closer. It is based on the assumption that hardly credible individual will need more advice than individuals with high credibility.

This study is structured in a bottom-up way. In Section 2, our proposal for a new consensus reaching process is presented. An example of application is reported in Section 3. We compare our approach with the existing ones in Section 4. Finally, some conclusions are offered in Section 5.

\section{Consensus reaching process based on individuals' credibility}

We introduce a consensus reaching process composed of three steps: (i) elicitation of evaluations, (ii) consensus control process, and (iii) feedback mechanism. It presents two main novelties: (i) in addition to the evaluations, the individuals provide some arguments to support them, and (ii) the feedback mechanism is based on the individuals' credibility in such a way that it will provide more advice to the individuals with lower credibility than to the individuals with higher credibility. The above three steps are detailed described in the following three subsections.

\subsection{Elicitation of evaluations}

We suppose that a fuzzy preference relation models the evaluations given by an individual. We use it by virtue of its ability to model decision processes and its usefulness and capacity to aggregate individual evaluations into group ones [13,14].

Definition 1 A fuzzy preference relation $P$ on a finite set $X$ is a fuzzy set on the Cartesian product $X \times X$, that $i s$, it is determined by means of a membership function $\mu_{P}: X \times X \rightarrow[0,1]$ over the set $X \times X$.

A fuzzy preference relation $P$ is commonly modeled by a $n \times n$ matrix $P=\left(p_{i j}\right)$ [14], being $p_{i j}=$ $\mu_{P}\left(x_{i}, x_{j}\right)$ the degree in which the option $x_{i}$ is preferred to the option $x_{j}$. In this setting, $p_{i j}=0.5$ means indifference between both options $\left(x_{i} \sim x_{j}\right), p_{i j}=1$ means that the option $x_{i}$ is entirely preferred to the option $x_{j}$, and $p_{i j}>0.5$ means that the option $x_{i}$ is preferred to the option $x_{j}\left(x_{i} \succ x_{j}\right)$. Furthermore, the elements of the main diagonal, that is, $p_{i i}$, are usually written as '- ' because they are not important here [13].

In addition to the elicitation of a fuzzy preference relation, each individual provides some arguments to justify her/his opinions. In particular, an argument $A r g_{i}^{k}$ is provided by each individual $e^{k}$ for each option $x_{i}$.

\subsection{Consensus control process}

Once all the individuals have expressed their evaluations, we need to obtain the consensus achieved. To do so, we follow the strategy usually employed when preference relations are used [15], which obtains three different consensus degrees. The methodology to obtain these consensus degrees is as follows:

- We define a similarity matrix, $S M^{k l}=\left(s m_{i j}^{k l}\right)$, for each pair of individuals $\left(e^{k}, e^{l}\right)$ as follows:

$$
s m_{i j}^{k l}=1-\left|p_{i j}^{k}-p_{i j}^{l}\right|
$$

- We aggregate all the similarity matrices to calculate a consensus matrix, $C M=\left(\mathrm{cm}_{i j}\right)$. To do so, we use, as aggregation function $\phi$, the arithmetic mean:

$$
c m_{i j}=\phi\left(s m_{i j}^{k l}\right)
$$

- We obtain the three consensus degrees:

1. Consensus degree on pairs of options, $c p_{i j}$, measuring the agreement achieved by the individuals on the pair formed by the options $x_{i}$ and $x_{j}$. The entry $(i, j)$ of $C M$ identifies this consensus degree:

$$
c p_{i j}=c m_{i j}
$$

2. Consensus degree on options, $c a_{i}$, measuring the agreement achieved by the individuals on the option $x_{i}$. It is computed as follows:

$$
c a_{i}=\frac{\sum_{j=1 ; j \neq i}^{n}\left(c p_{i j}+c p_{j i}\right)}{2(n-1)}
$$

3. Consensus degree on the relation, $c r$, measuring the global agreement achieved by the individuals. It is calculated as follows:

$$
c r=\frac{\sum_{i=1}^{n} c a_{i}}{n}
$$

The value of $\mathrm{cr}$ controls the consensus reaching process. Its value is contrasted with a minimum consensus threshold, $\alpha \in[0,1]$, whose value depends on the problem under study [7]. On the one hand, if $\mathrm{cr}<\alpha$, the feedback mechanism is applied with the purpose of modifying the evaluations given by the individuals and, as a consequence, to increase the value of $c r$. On the other hand, if $c r \geq \alpha$, the selection process is carried out 
to rank the options from best to worst. In addition, with the purpose of avoiding a situation in which $\mathrm{cr}$ does not converge to $\alpha$, a maximum number of discussion rounds is fixed [6]. Then, if the number of the current discussion round exceeds it, the selection process is also applied.

\subsection{Feedback mechanism}

On the assumption that the individuals with a low knowledge level of the problem will need more recommendations than individuals with a high knowledge level, the feedback mechanism proposed here tries to adapt its performance to the different kinds of individuals. We suppose that individuals with a high credibility provide evaluations belong to a wider knowledge than the remaining individuals. Therefore, a few modifications in their evaluations might lead to consensus. On the other hand, individuals with a low credibility will require many modifications in their evaluations to lead a consensual solution.

To accomplish this task, each individual is included by her/his credibility degree into one of the three following subsets: (i) very credible, $E_{v c}$, (ii) credible, $E_{c}$, and (iii) hardly credible, $E_{h d}$. The credibility degree is interpreted as a fuzzy subset, Cred, with a membership function, $\mu_{C r e d}: E \rightarrow[0,1]$, in such a way that $\mu_{C r e d}\left(e^{k}\right) \in[0,1]$ denotes the credibility degree of the evaluations given by the individual $e^{k}$. Then, a fuzzy matching mechanism, whose parameters depend on the problem at hand, is applied to done this classification. Each subset of individuals is a fuzzy set characterized by a membership function and two membership thresholds, $\beta_{1}$ and $\beta_{2}$, are established to differentiate the subsets.

Given a particular individual, $e^{k}$, her/his credibility degree, $\mu_{C r e d}\left(e^{k}\right)$, is computed as follows:

$$
\mu_{C r e d}\left(e^{k}\right)=\frac{\sum_{l=1 ; l \neq k}^{m} \mu_{C r e d}\left(e_{l}^{k}\right)}{m-1}
$$

being $\mu_{C r e d}\left(e_{l}^{k}\right) \in[0,1]$ the credibility degree assigned by the individual $e^{l}$ to the individual $e^{k}$ after reading the arguments provided by the individual $e^{k}$ to justify her/his evaluations.

In what follows, we describe in detail this feedback mechanism, which is composed of three steps: (i) computing proximity measures, (ii) searching for controversial evaluations, and (iii) advising.

2.3.1. Computing proximity measures Similar to the existing approaches [3, 6, 7], the feedback mechanism calculates first the level of agreement between the individual evaluations and the group ones. To do this, proximity measures are computed as follows:
- We aggregate all the fuzzy preference relations to compute a collective matrix, $P^{c}=\left(p_{i j}^{c}\right)$ :

$$
p_{i j}^{c}=\Phi\left(p_{i j}^{1}, p_{i j}^{2}, \ldots, p_{i j}^{m}\right)
$$

The aggregation function, $\Phi$, should consider that the individuals have different credibility degrees. In an aggregation process, the procedure for considering weighted values involves the transformation of the values under the weight to produce a new value [16]. Then, these new values are aggregated using an aggregation function. Here, we use the weighted average to consider the credibility degrees, which act as weights of the evaluation:

$$
p_{i j}^{c}=\frac{\sum_{k=1}^{m} \mu_{C r e d}\left(e^{k}\right) \cdot p_{i j}^{k}}{\sum_{k=1}^{m} \mu_{C r e d}\left(e^{k}\right)}
$$

- Once we have calculated the collective matrix, we compute the proximity measures:

1. Proximity measure on pairs of options, $p p_{i j}^{k}$, measuring the similarity between the evaluation given by the individual $e^{k}$ on a pair of options $\left(x_{i}, x_{j}\right)$ and the collective one:

$$
p p_{i j}^{k}=1-\left|p_{i j}^{k}-p_{i j}^{c}\right|
$$

2. Proximity measure on options, $p a_{i}^{k}$, measuring the similarity between the evaluation given by the individual $e^{k}$ on the option $x_{i}$ and the collective one:

$$
p a_{i}^{k}=\frac{\sum_{j=1, j \neq i}^{n}\left(p p_{i j}^{k}+p p_{j i}^{k}\right)}{2(n-1)}
$$

3. Proximity measure on the relation, $p r^{k}$, measuring the global similarity between the evaluations of the individual $e^{k}$ and the collective one:

$$
p r^{k}=\frac{\sum_{i=1}^{n} p a_{i}^{k}}{n}
$$

2.3.2. Searching for controversial evaluations The second step consists of identifying the evaluations that should be modified to increase the agreement in the next discussion round.

The proposed feedback mechanism is composed of three different strategies identifying the evaluations that the individuals should change. That is, each one of 
the previous subsets entails a different mechanism to determine the controversial evaluations (those with low agreement).

1. Identifying controversial evaluations for hardly credible individuals. It seems reasonable that, a priori, the individuals located in this subset may express less informed evaluations. As a consequence, more changes should be required here to increase the consensus. Therefore, the strategy applied to this subset tries to change the evaluations on all the pairs of options in which the agreement is low. It acts as follows:

(a) A threshold, $\gamma_{1}$, is determined as the average of the consensus degrees at the level of all pairs of options:

$$
\gamma_{1}=\sum_{i=1}^{n}\left(\sum_{j=1, j \neq i}^{n} c p_{i j}\right) /\left(n^{2}-n\right)
$$

(b) Identifying the pairs of options, $P O$, whose consensus degree is smaller than the threshold $\gamma_{1}$ :

$$
P O=\left\{\left(x_{i}, x_{j}\right) \mid c p_{i j}<\gamma_{1}\right\}
$$

(c) Identifying the controversial evaluations, $C E_{h c}^{k}$, that each individual $e^{k} \in E_{h c}$ should change:

$$
C E_{h c}^{k}=P O
$$

2. Identifying controversial evaluations for credible individuals. In this case, it is reasonable to change the strategy applied by reducing the number of modifications. Instead of focusing on all the pairs of options in disagreement, this strategy analyzes the agreement from the point of view of the options and only considers the evaluations in disagreement of the options in which the agreement is low. In addition, the previous strategy requires that all individuals change the identified evaluations. However, here, for the identified options in disagreement, only the individuals whose proximity measure at level of options is smaller than a proximity threshold $\delta_{1}$ are suggested to change their evaluations. This strategy identifies the evaluations to be modified by each individual, $e^{k} \in E_{c}$, as follows:

(a) A threshold, $\gamma_{2}$, is determined as the average of the consensus degrees at level of options:

$$
\gamma_{2}=\sum_{i=1}^{n} c a_{i} / n
$$

(b) Identifying the options, $O$, to be modified:

$$
O=\left\{x_{i} \mid c a_{i}<\gamma_{2}\right\}
$$

(c) Identifying the pairs of options, $P O$, to be modified:

$$
P O=\left\{\left(x_{i}, x_{j}\right) \mid x_{i} \in O \wedge c p_{i j}<\gamma_{1}\right\}
$$

(d) A threshold $\delta_{1}$, for each option $x_{i} \in O$, is determined as follows:

$$
\delta_{1}=\sum_{k=1}^{m} p a_{i}^{k} / m, e^{k} \in E_{c}
$$

(e) Identifying the controversial evaluations, $C E_{c}^{k}$, that should be changed:

$$
C E_{c}^{k}=\left\{\left(x_{i}, x_{j}\right) \in P O \mid p a_{i}^{k}<\delta_{1}\right\}
$$

3. Identifying controversial evaluations for very credible individuals. This strategy deals with the individuals located in the subset $E_{v c}$, that is, the individuals whose credibility is so high that they do not need to be strongly changed to obtain a well-considered evaluation. Hence, fewer modifications than in the previous two strategies should be suggested here to improve the agreement. In particular, this strategy only modifies, for the pairs of options identified in disagreement, the evaluations of the individuals whose proximity measure is smaller than a proximity threshold at the level of pair of options, $\delta_{2}$. This strategy identifies the evaluations to be modified by each individual, $e^{k} \in E_{v c}$, as follows:

(a) Identifying the options, $O$, to be modified:

$$
O=\left\{x_{i} \mid c a_{i}<\gamma_{2}\right\}
$$

(b) Identifying the pairs of options, $P O$, to be modified:

$$
P O=\left\{\left(x_{i}, x_{j}\right) \mid x_{i} \in O \wedge c p_{i j}<\gamma_{1}\right\}
$$

(c) A threshold $\delta_{2}$, for each pair of options $\left(x_{i}, x_{j}\right) \in P O$, is determined as follows:

$$
\delta_{2}=\sum_{k=1}^{m} p p_{i j}^{k} / m, e^{k} \in E_{v c}
$$

(d) Identifying the controversial evaluations, $C E_{v c}^{k}$, that are required to be modified:

$$
\begin{gathered}
C E_{v c}^{k}=\left\{\left(x_{i}, x_{j}\right) \in P O \mid p a_{i}^{k}<\delta_{1} \wedge\right. \\
\left.\wedge p p_{i j}^{k}<\delta_{2}\right\}
\end{gathered}
$$


2.3.3. Advising Once the controversial evaluations have been identified, the next step consists in showing the right direction of the modifications. Similar to other approaches [7], we apply a mechanism based on two direction rules. Then, for each evaluation identified as controversial, the feedback mechanism suggests the modification of the current evaluation as follows:

- The evaluation given by the individual $e^{k}$ on the pair $x_{i}$ and $x_{j}$ must be increased if $p_{i j}^{k}<p_{i j}^{c}$.

- The evaluation given by the individual $e^{k}$ on the pair $x_{i}$ and $x_{j}$ must be decreased if $p_{i j}^{k}>p_{i j}^{c}$.

\section{Illustrative example}

Let us suppose that a school wants to improve some of its services by investing some money. These services are: $x_{1}=$ Cafeteria, $x_{2}=$ Library, $x_{3}=$ Website, and $x_{4}=$ Sport court. The aim is to distribute the money among the services according to the necessity of improving each one of them. In consequence, the money received by each service is related to its position in the ranking: the best position in the ranking is, the more money it receives. To accomplish this task, four individuals (users and school staff) are inquired.

The proposed approach is set up as follows: $\alpha$ is equal to 0.75 , the maximum number of discussion rounds is equal to 5 , and the membership thresholds, $\beta_{1}$ and $\beta_{2}$ are equal to 0.65 and 0.45 , respectively.

In what follows, we apply our proposal to solve this problem. In the first discussion round, we describe all the steps in detail. In the next discussion rounds, we only show the most relevant information: the new evaluations given by the individuals, the evolution of the consensus and the advice generated by the feedback mechanism.

\subsection{First discussion round}

3.1.1. Elicitation of evaluations Initially, the individuals involved in the problem provide the following fuzzy preference relations:

$$
\begin{aligned}
P^{1} & =\left(\begin{array}{cccc}
- & 0.90 & 0.90 & 0.90 \\
0.10 & - & 0.70 & 0.80 \\
0.20 & 0.30 & - & 0.50 \\
0.20 & 0.20 & 0.50 & -
\end{array}\right) \\
P^{2} & =\left(\begin{array}{cccc}
- & 0.10 & 0.30 & 0.30 \\
0.90 & - & 0.80 & 0.90 \\
0.70 & 0.20 & - & 0.50 \\
0.80 & 0.10 & 0.50 & -
\end{array}\right)
\end{aligned}
$$

$$
\begin{aligned}
P^{3} & =\left(\begin{array}{cccc}
- & 0.50 & 0.10 & 0.30 \\
0.50 & - & 0.20 & 0.20 \\
0.90 & 0.80 & - & 0.50 \\
0.80 & 0.90 & 0.50 & -
\end{array}\right) \\
P^{4} & =\left(\begin{array}{cccc}
- & 0.50 & 0.30 & 0.30 \\
0.50 & - & 0.20 & 0.20 \\
0.80 & 0.80 & - & 0.50 \\
0.70 & 0.70 & 0.50 & -
\end{array}\right)
\end{aligned}
$$

Additionally, they express these arguments that make them to have the previous evaluations:

$\operatorname{Arg}_{1}^{1}=$ Good quality of the food.

$\operatorname{Arg}_{2}^{1}=$ Old Furniture.

$\operatorname{Arg}_{3}^{1}=$ Modern and dynamic.

$\mathrm{Arg}_{4}^{1}=$ Incorporate more sports.

$\operatorname{Arg}_{1}^{2}=$ Need to improve the service.

$\operatorname{Arg}_{2}^{2}=$ Need more staff.

$\operatorname{Arg}_{3}^{2}=$ Should include more features.

$\mathrm{Arg}_{4}^{2}=\mathrm{It}$ is almost new.

$A r g_{1}^{3}=$ Old furniture and materials.

$\operatorname{Arg}_{2}^{3}=$ Small study room.

$\mathrm{Arg}_{3}^{3}=$ Enough for the students.

$\mathrm{Arg}_{4}^{3}=$ More chairs for spectators.

$\operatorname{Arg}_{1}^{4}=$ Bad air conditioning system.

$\operatorname{Arg}_{2}^{4}=$ Good electronic resources.

$\operatorname{Arg}_{3}^{4}=$ Should include more teacher resources.

$\mathrm{Arg}_{4}^{4}=$ Enough for school sport teams.

3.1.2. Consensus control process First, we compute the six similarity matrices, one for each pair of individuals (they are omitted). Then, we calculate the $C M$, whose entries are used to obtain the different consensus degrees:

1. Consensus degrees on pairs of options:

$$
C M=\left(\begin{array}{cccc}
- & 0.60 & 0.60 & 0.70 \\
0.60 & - & 0.62 & 0.55 \\
0.63 & 0.62 & - & 1.00 \\
0.68 & 0.52 & 1.00 & -
\end{array}\right)
$$

2. Consensus degree on options:

$$
\begin{array}{ll}
c a_{1}=0.64 & c a_{2}=0.58 \\
c a_{3}=0.75 & c a_{4}=0.74
\end{array}
$$


3. Consensus degree on the relation:

$$
c r=0.68
$$

After computing the consensus degrees, $\mathrm{cr}$ is equal to 0.68. Because this value is lower than the minimum consensus threshold $(\alpha=0.75)$, and the current discussion round is the first one, the feedback mechanism is activated.

3.1.3. Feedback mechanism The first time the feedback mechanism is activated, the individuals are located in one of the three different subsets considering their credibility. Each individual reads the arguments given by the others and assigns a credibility degree to them. Once each individual has assigned a credibility degree to each one of the others, the final credibility degree obtained by each individual using (6) is:

$$
\begin{array}{ll}
\mu_{\text {Cred }}\left(e^{1}\right)=0.70 & \mu_{C r e d}\left(e^{2}\right)=0.50 \\
\mu_{\text {Cred }}\left(e^{3}\right)=0.40 & \mu_{\text {Cred }}\left(e^{4}\right)=0.40
\end{array}
$$

To compute the customized recommendations, the individuals are included by their credibility degree into three different subsets (taking $\beta_{1}$ and $\beta_{2}$ as reference):

$$
E_{h c}=\left\{e^{3}, e^{4}\right\} \quad E_{c}=\left\{e^{2}\right\} \quad E_{v c}=\left\{e^{1}\right\}
$$

- Computing proximity measures: First the collective fuzzy preference relation is calculated by aggregating the four individual fuzzy preference relations using (8):

$$
P^{c}=\left(\begin{array}{cccc}
- & 0.54 & 0.47 & 0.51 \\
0.46 & - & 0.52 & 0.58 \\
0.58 & 0.47 & - & 0.50 \\
0.57 & 0.41 & 0.50 & -
\end{array}\right)
$$

1. Proximity measures on pairs of options:

$$
\begin{aligned}
P P^{1} & =\left(\begin{array}{cccc}
- & 0.64 & 0.57 & 0.61 \\
0.64 & - & 0.82 & 0.78 \\
0.61 & 0.82 & - & 1.00 \\
0.63 & 0.78 & 1.00 & -
\end{array}\right) \\
P P^{2} & =\left(\begin{array}{cccc}
- & 0.56 & 0.83 & 0.79 \\
0.56 & - & 0.72 & 0.68 \\
0.88 & 0.72 & - & 1.00 \\
0.77 & 0.68 & 1.00 & -
\end{array}\right) \\
P P^{3} & =\left(\begin{array}{cccc}
- & 0.96 & 0.63 & 0.79 \\
0.96 & - & 0.67 & 0.61 \\
0.68 & 0.67 & - & 1.00 \\
0.77 & 0.51 & 1.00 & -
\end{array}\right)
\end{aligned}
$$

$$
P P^{4}=\left(\begin{array}{cccc}
- & 0.96 & 0.83 & 0.79 \\
0.96 & - & 0.67 & 0.61 \\
0.78 & 0.67 & - & 1.00 \\
0.87 & 0.71 & 1.00 & -
\end{array}\right)
$$

2. Proximity measures on options:

$$
\begin{array}{llll}
p a_{1}^{1}=0.62 & p a_{2}^{1}=0.75 & p a_{3}^{1}=0.80 & p a_{4}^{1}=0.80 \\
p a_{1}^{2}=0.73 & p a_{2}^{2}=0.66 & p a_{3}^{2}=0.86 & p a_{4}^{2}=0.82 \\
p a_{1}^{3}=0.80 & p a_{2}^{3}=0.73 & p a_{3}^{3}=0.78 & p a_{4}^{3}=0.78 \\
p a_{1}^{4}=0.86 & p a_{2}^{4}=0.77 & p a_{3}^{4}=0.83 & p a_{4}^{4}=0.83
\end{array}
$$

3. Proximity measures on the relation:

$$
\begin{array}{ll}
p r^{1}=0.74 & p r^{3}=0.77 \\
p r^{3}=0.77 & p r^{4}=0.82
\end{array}
$$

\section{- Searching for controversial evaluations:}

1. Identifying controversial evaluations for hardly credible individuals:

(a) The threshold $\gamma_{1}$ is equal to 0.68 .

(b) Pairs of options whose consensus degree is smaller than the threshold $\gamma_{1}$ :

$$
\begin{gathered}
P O=\left\{\left(x_{1}, x_{2}\right),\left(x_{1}, x_{3}\right),\left(x_{2}, x_{1}\right),\right. \\
\left(x_{2}, x_{3}\right),\left(x_{2}, x_{4}\right),\left(x_{3}, x_{1}\right), \\
\left.\left(x_{3}, x_{2}\right),\left(x_{4}, x_{2}\right)\right\}
\end{gathered}
$$

(c) Evaluations to be changed by individuals $e^{3}$ and $e^{4}$ :

$$
\begin{aligned}
& C E_{h c}^{3}=P O \\
& C E_{h c}^{4}=P O
\end{aligned}
$$

2. Identifying controversial evaluations for credible individuals:

(a) The threshold $\gamma_{2}$ is equal to 0.68 .

(b) Options in which the consensus degree is not high enough:

$$
O=\left\{x_{1}, x_{2}\right\}
$$

(c) Pair of options in disagreement:

$$
\begin{gathered}
P O=\left\{\left(x_{1}, x_{2}\right),\left(x_{1}, x_{3}\right),\left(x_{2}, x_{1}\right),\right. \\
\left.\left(x_{2}, x_{3}\right),\left(x_{2}, x_{4}\right)\right\}
\end{gathered}
$$

(d) The threshold $\delta_{1}$ is equal to 0.75 for the option $x_{1}$ and equal to 0.73 for the option $x_{2}$. 
(e) Evaluations to be changed by the individual $e^{2}$ :

$$
\begin{aligned}
C E_{c}^{2}=\left\{\left(x_{1}, x_{2}\right),\left(x_{1}, x_{3}\right),\left(x_{2}, x_{1}\right),\right. \\
\left.\left(x_{2}, x_{3}\right),\left(x_{2}, x_{4}\right)\right\}
\end{aligned}
$$

3. Identifying controversial evaluations for very credible individuals:

(a) Options whose consensus degree is not high enough:

$$
O=\left\{x_{1}, x_{2}\right\}
$$

(b) Pair of options in disagreement:

$$
\begin{gathered}
P O=\left\{\left(x_{1}, x_{2}\right),\left(x_{1}, x_{3}\right),\left(x_{2}, x_{1}\right),\right. \\
\left.\left(x_{2}, x_{3}\right),\left(x_{2}, x_{4}\right)\right\}
\end{gathered}
$$

(c) The threshold $\delta_{2}$ is computed as the average of the proximity measures on pairs of options of those pairs of options identified to be modified previously.

(d) Evaluations to be changed by the individual $e^{1}$ :

$$
C E_{h c}^{1}=\left\{\left(x_{1}, x_{2}\right),\left(x_{1}, x_{3}\right)\right\}
$$

- Advising: The feedback mechanism suggests the modification of the current evaluations according to the following direction rules (the symbol + means that this evaluation should be increased, and the symbol - means that this evaluation should be decreased):

$$
\begin{gathered}
P^{1}=\left(\begin{array}{llll}
= & - & = & = \\
= & = & = \\
= & = & =
\end{array}\right) P^{2}=\left(\begin{array}{cccc}
- & + & = \\
- & - & - \\
= & = & = \\
= & = & =
\end{array}\right) \\
P^{3}=\left(\begin{array}{lll}
+ & + & = \\
- & + & + \\
= & = & =
\end{array}\right) P^{4}=\left(\begin{array}{lll}
- & + & = \\
- & + & + \\
- & = & =
\end{array}\right)
\end{gathered}
$$

\subsection{Second discussion round}

3.2.1. Elicitation of evaluations In this discussion round, we assume that the individuals agree with the recommendations produced by the feedback mechanism. In such a way, the new fuzzy preference relations are:

$$
P^{1}=\left(\begin{array}{cccc}
- & \mathbf{0 . 8 0} & \mathbf{0 . 8 0} & 0.90 \\
0.10 & - & 0.70 & 0.80 \\
0.20 & 0.30 & - & 0.50 \\
0.20 & 0.20 & 0.50 & -
\end{array}\right)
$$

$$
\begin{aligned}
P^{2} & =\left(\begin{array}{cccc}
- & \mathbf{0 . 2 0} & \mathbf{0 . 4 0} & 0.30 \\
\mathbf{0 . 8 0} & - & \mathbf{0 . 7 0} & \mathbf{0 . 8 0} \\
0.70 & 0.20 & - & 0.50 \\
0.80 & 0.10 & 0.50 & -
\end{array}\right) \\
P^{3} & =\left(\begin{array}{cccc}
- & \mathbf{0 . 6 0} & \mathbf{0 . 2 0} & 0.30 \\
\mathbf{0 . 4 0} & - & \mathbf{0 . 3 0} & \mathbf{0 . 3 0} \\
\mathbf{0 . 8 0} & \mathbf{0 . 7 0} & - & 0.50 \\
0.80 & \mathbf{0 . 8 0} & 0.50 & -
\end{array}\right) \\
P^{4} & =\left(\begin{array}{cccc}
- & \mathbf{0 . 6 0} & \mathbf{0 . 4 0} & 0.30 \\
\mathbf{0 . 4 0} & - & \mathbf{0 . 3 0} & \mathbf{0 . 3 0} \\
\mathbf{0 . 7 0} & \mathbf{0 . 7 0} & - & 0.50 \\
0.70 & \mathbf{0 . 6 0} & 0.50 & -
\end{array}\right)
\end{aligned}
$$

3.2.2. Consensus control process After computing the consensus degrees using these new fuzzy preference relations, the $c r$ is equal to 0.73 . Now, the consensus is higher than in the previous discussion round. However, it is still lower than $\alpha=0.75$. In addition, the current discussion round is the second one. Therefore, the feedback mechanism is activated.

3.2.3. Feedback mechanism After computing the proximity measures and identifying the controversial evaluations, the feedback mechanism suggests the modification of the current evaluations according to the following direction rules:

$$
\begin{gathered}
P^{1}=\left(\begin{array}{llll}
= & - & - \\
= & = & = \\
= & = & =
\end{array}\right) P^{2}=\left(\begin{array}{lll}
- & + & + \\
= & = & - \\
= & = & = \\
= & = &
\end{array}\right) \\
P^{3}=\left(\begin{array}{lll}
- & + & + \\
- & = & + \\
- & - & =
\end{array}\right) P^{4}=\left(\begin{array}{lll}
- & + & + \\
- & = & + \\
- & = & =
\end{array}\right)
\end{gathered}
$$

\subsection{Third discussion round}

3.3.1. Elicitation of preferences In the third discussion round, we assume again that the individuals agree with the recommendations returned by the feedback mechanism. Now, the new fuzzy preference relations are:

$$
P^{1}=\left(\begin{array}{cccc}
- & \mathbf{0 . 7 0} & \mathbf{0 . 7 0} & \mathbf{0 . 8 0} \\
0.10 & - & 0.70 & 0.80 \\
0.20 & 0.30 & - & 0.50 \\
0.20 & 0.20 & 0.50 & -
\end{array}\right)
$$




$$
\begin{aligned}
P^{2} & =\left(\begin{array}{cccc}
- & \mathbf{0 . 3 0} & \mathbf{0 . 5 0} & \mathbf{0 . 4 0} \\
\mathbf{0 . 7 0} & - & 0.60 & \mathbf{0 . 8 0} \\
0.70 & 0.20 & - & 0.50 \\
0.80 & 0.10 & 0.50 & -
\end{array}\right) \\
P^{3} & =\left(\begin{array}{cccc}
- & \mathbf{0 . 5 0} & \mathbf{0 . 3 0} & \mathbf{0 . 4 0} \\
\mathbf{0 . 3 0} & - & 0.30 & \mathbf{0 . 4 0} \\
\mathbf{0 . 7 0} & \mathbf{0 . 6 0} & - & 0.50 \\
\mathbf{0 . 7 0} & \mathbf{0 . 7 0} & 0.50 & -
\end{array}\right) \\
P^{4} & =\left(\begin{array}{cccc}
- & \mathbf{0 . 5 0} & \mathbf{0 . 5 0} & \mathbf{0 . 4 0} \\
\mathbf{0 . 3 0} & - & 0.30 & \mathbf{0 . 4 0} \\
\mathbf{0 . 6 0} & \mathbf{0 . 6 0} & - & 0.50 \\
\mathbf{0 . 6 0} & \mathbf{0 . 5 0} & 0.50 & -
\end{array}\right)
\end{aligned}
$$

3.3.2. Consensus control process After computing the consensus degrees using these new fuzzy preference relations, the $\mathrm{cr}$ is equal to 0.78 . Now, the consensus is higher than in the second discussion round and also higher than $\alpha=0.75$. Therefore, the selection process is applied to rank the options from best to worst.

\subsection{Selection process}

The selection process ranks the options from best to worst according to the individuals' evaluations. Since the objective of this study is not to present a new selection process, if the reader is interesting in knowing more about it, see [4]. It consists of two phases: (i) aggregation, which obtains a collective preference relation, and (ii) exploitation, which uses the collective preference relation to rank the options.

In the aggregation phase, (8) is used to obtain the collective fuzzy preference relation:

$$
P^{c}=\left(\begin{array}{cccc}
- & 0.52 & 0.53 & 0.54 \\
0.33 & - & 0.51 & 0.64 \\
0.50 & 0.39 & - & 0.50 \\
0.53 & 0.33 & 0.50 & -
\end{array}\right)
$$

At this point, in order to transform the global preference about the options into a ranking of them, the quantifier-guided dominance degree, $Q G D D_{i}$, quantifying the dominance that the option $x_{i}$ has over all the others is employed [4]:

$$
Q G D D_{i}=\phi\left(p_{i 1}^{c}, \ldots, p_{i(i-1)}^{c}, p_{i(i+1)}^{c}, \ldots, p_{i n}^{c}\right)
$$

Using the average as aggregation function, $\phi$, the ranking obtained is: $x_{1} \succ x_{2} \succ x_{3} \succ x_{4}$.

\section{Discussion}

In this section, we compare the consensus reaching process presented in this paper with other approaches existing in the literature and analyze its convergence.

- Consensus reaching processes incorporating a feedback mechanism. Different approaches have been proposed $[6,17,18]$ in which the feedback mechanism incorporated is considered as an inflexible or rigid one since its performance remains fixed during all the discussion rounds. As in these approaches the individuals are considered equally important, to compare the proposed approach with them, we use our approach, but substituting both its searching for controversial evaluation step and its advising step by the feedback mechanism presented in those approaches. Using the same data as in the example illustrated in Section 3, the consensus is reached in the third round, being $c r=0.79$. The ranking of options obtained using the selection process is $x_{2} \succ x_{3} \succ x_{4} \succ x_{1}$, which is quite different to the ranking obtained using our approach. According to the first evaluations given by the individuals, it may be seen that the best options should be $x_{1}$ and $x_{2}$ as they are the preferred by the individuals $e^{1}$ and $e^{2}$, which have the higher credibility degrees. Therefore, they should be the leaders of the discussion. This situation occurs in our approach, as the final ranking of options obtained is $x_{1} \succ x_{2} \succ x_{3} \succ$ $x_{4}$. However, if the feedback mechanism does not take into account the individuals' credibility (knowledge level or importance level) to generate the recommendations, a situation, which is not desired, may happen. Therefore, in this case, the options $x_{3}$ and $x_{4}$, which were the most preferred by the individuals $e^{3}$ and $e^{4}$, are preferred to the option $x_{1}$. The reason of it is that, although the individuals $e^{3}$ and $e^{4}$ have a lower credibility, the addition of their evaluations, which are similar, has made them important as a group and they have become the leaders of the discussion. It is due to the feedback mechanism considers all individuals with equal credibility (knowledge level or importance level), generating the same amount of advice for them. As a result, to increase the consensus level, the most credible individual, $e^{1}$, has to change her/his evaluations according to the individuals $e^{3}$ and $e^{4}$. However, as the proposed feedback mechanism generates recommendations according to the individuals' credibility, the lower credible individuals receive 
more advice to change their evaluations, allowing for the individuals with more credibility to be the leaders of the discussion and convince the other individuals to modify their evaluations to improve the agreement. On the other hand, the consensus reaching process presented in [7] presents the same problem. In this case, its feedback mechanism adapts its performance to the consensus reached in each discussion round. This reduces the number of discussion rounds and, therefore, the convergence to the consensus is faster. However, as it does not take into account the individuals' credibility to generate the advice, the same problem shown in the above approaches appears here. Finally, the approach presented in [1] is the most similar to the approach proposed in this study, but adapting the computed amount of advice to the individuals' importance. However, we incorporate the arguments in order to go one step further obtaining credibility degrees instead of just weight values, which are subjectively assigned at the beginning of the process.

- Group decision making approaches using the heterogeneity of the individuals in the selection process [9, 16, 19]. The main drawback of applying a selection process directly, without a consensus reaching process, is that some individuals could think that their evaluations have not properly considered to obtain the rank of options and, as a consequence, these individuals could reject the solution obtained. In this way, in the example illustrated in Section 3, if we apply the selection process directly, the ranking of options obtained is $x_{2} \succ x_{3} \succ x_{1} \succ x_{4}$, being the consensus reached equal to 0.68 , which is low. Moreover, as in these approaches there is no discussion, if there are some low credible individuals, but whose evaluations are similar, they could become the main leaders instead of the most credible individuals. For instance, in this example, the option $x_{1}$, which should be the best one according to the most credible individual, $e^{1}$, is ranked in the third place.

- In the proposed approach, since the consistency associated with the fuzzy preference relations is not checked, inconsistent evaluations could be provided. As a result, misleading rankings could be obtained. Therefore, a mechanism to control and improve the consistency associated with the fuzzy preference relations provided by the individuals should be added to the proposed approach $[19,20]$.

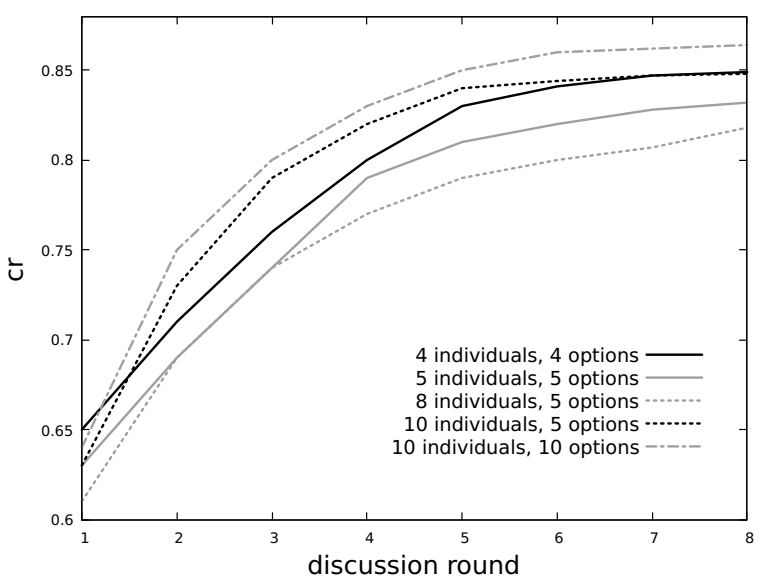

Figure 1. $c r$ in successive discussion rounds.

- To analyze the convergence of the proposed approach, we simulate various decision problems with different sets of individuals and options (in particular, 8 discussion rounds are simulated). If the individuals accept the advice provided by the feedback mechanism, the consensus achieved in each discussion round is increased (see Fig. 1). Thus, the proposed approach converges to solutions with higher consensus. Anyway, if the minimum consensus threshold is not achieved because the individuals do not follow the advice generated or it would require many discussion rounds to be achieved, a maximum number of discussion rounds is usually fixed [6].

\section{Conclusions}

We have introduced a novel feedback mechanism, which considers the individuals' credibility, as part of a consensus reaching process. Assuming different types of individuals classified according to their credibility the feedback mechanism customizes the advice generated. To do this, it presents three strategies to recognize the controversial evaluations that the individuals must modify if they want to increase the consensus. In every discussion round, these three strategies are carried out in a different way based on the individuals' credibility by considering both the current consensus degrees and the proximity measures calculated. The aim is that the evaluations of the most credible individuals never be strongly changed throughout the discussion rounds. It allows for the most credible individuals to be the leaders of the discussion and convince the other individuals to modify their evaluations to improve the agreement. In addition, as a new step in the consensus reaching process, the individuals have to express the arguments 
that justify their evaluations. This helps classify the individuals into one of three different subsets (very credible, credible and hardly credible).

This new approach may be applied to solve different real world problems as, for instance, water allocation management [21], credit risk evaluation [22], e-democracy [23], and so on. Finally, as future work, we propose that this new approach may provide several rankings, each of them with a degree of reliability.

\section{Acknowledgments}

The work was supported by the Grant from the FEDER funds provided by the Spanish Ministry of Economy and Competitiveness (No. TIN2016-75850-R) and by the Programa de Fomento e Impulso de la actividad investigadora de la Universidad de Cádiz.

\section{References}

[1] I. J. Pérez, F. J. Cabrerizo, S. Alonso, and E. Herrera-Viedma, "A new consensus model for group decision making problems with non homogeneous experts," IEEE Transactions on Systems, Man, and Cybernetics: Systems, vol. 44, no. 4, pp. 494-498, 2014.

[2] C. T. Butler and A. Rothstein, On conflict and consensus: A handbook on formal consensus decisionmaking. Portland, Maine: Food Not Bombs Publishing, 1987.

[3] E. Herrera-Viedma, F. J. Cabrerizo, J. Kacprzyk, and W. Pedrycz, "A review of soft consensus models in a fuzzy environment," Information Fusion, vol. 17, pp. 4-13, 2014.

[4] F. J. Cabrerizo, R. Heradio, I. J. Pérez, and E. Herrera-Viedma, "A selection process based on additive consistency to deal with incomplete fuzzy linguistic information," Journal of Universal Computer Science, vol. 16, no. 1, pp. 62-81, 2010.

[5] J. Kacprzyk, M. Fedrizzi, and H. Nurmi, "Group decision making and consensus under fuzzy preferences and fuzzy majority," Fuzzy Sets and Systems, vol. 49, no. 1, pp. 21-31, 1992.

[6] E. Herrera-Viedma, S. Alonso, F. Chiclana, and F. Herrera, "A consensus model for group decision making with incomplete fuzzy preference relations," IEEE Transactions on Fuzzy Systems, vol. 15, no. 5, pp. 863-877, 2007.

[7] F. Mata, L. Martínez, and E. Herrera-Viedma, "An adaptive consensus support model for group decision-making problems in a multigranular fuzzy linguistic context," IEEE Transactions on Fuzzy Systems, vol. 17, no. 2, pp. 279-290, 2009.

[8] A. Taghavi, E. Eslami, F. J. Cabrerizo, and E. Herrera-Viedma, "Analyzing feedback mechanisms in group decision making problems," in 10th Conference on the European Society for Fuzzy Logic and Technology, vol. 643 of Advances in Intelligent Systems and Computing, pp. 371-382, September 2017.

[9] R. R. Yager, "Weighted maximum entropy owa aggregation with applications to decision making under risk," IEEE Transactions on Systems, Man, and Cibernetics - Part A: Systems and Humans, vol. 39, no. 3, pp. 555-564, 2009.

[10] A. Gürkan and L. Iandoli, "Argument mapping and content fusion to support the analysis and synthesis of information in online discussions," International Journal of Decision Support System Technology, vol. 6, no. 1, pp. 14-33, 2014.

[11] L. Iandoli, I. Quinto, P. Spada, M. Klein, and R. Calabretta, "Supporting argumentation in online political debate: Evidence from an experiment of collective deliberation," New Media \& Society, vol. 20, no. 4, pp. 1320-1341, 2018.

[12] R. S. Arvapally and X. Liu, "Analyzing credibility of arguments in a web-based intelligent argumentation system for collective decision support based on k-means clustering algorithm," Knowledge Management Research \& Practice, vol. 10, no. 4, pp. 326-341, 2012.

[13] J. Kacprzyk, "Group decision making with a fuzzy linguistic majority," Fuzzy Sets and Systems, vol. 18, no. 2, pp. 105-118, 1986.

[14] T. Tanino, "Fuzzy preference orderings in group decision making," Fuzzy Sets and Systems, vol. 12, no. 2, pp. 117-131, 1984.

[15] R. Ureña, F. J. Cabrerizo, J. M. Morente-Molinera, and E. Herrera-Viedma, "GDM-R: A new framework in $\mathrm{R}$ to support fuzzy group decision making processes," Information Sciences, vol. 357, pp. 161-181, 2016.

[16] F. J. Montero de Juan, "Aggregation of fuzzy opinion in a non-homogeneous group," Fuzzy Sets and Systems, vol. 25, no. 1, pp. 15-20, 1987.

[17] F. J. Cabrerizo, I. J. Pérez, and E. Herrera-Viedma, "Managing the consensus in group decision making in an unbalanced fuzzy linguistic context with incomplete information," Knowledge-Based Systems, vol. 23, no. 3, pp. 169-181, 2010.

[18] E. Herrera-Viedma, F. Herrera, and F. Chiclana, "A consensus model for multiperson decision making with different preference structures," IEEE Transactions on Systems, Man, and Cybernetics - Part A: Systems and Humans, vol. 32, no. 3, pp. 394-402, 2002.

[19] F. J. Cabrerizo, E. Herrera-Viedma, and W. Pedrycz, "A method based on pso and granular computing of linguistic information to solve group decision making problems defined in heterogeneous context," European Journal of Operational Research, vol. 230, no. 3, pp. 624-633, 2013.

[20] F. Chiclana, F. Mata, L. Martínez, E. Herrera-Viedma, and S. Alonso, "Integration of a consistency control module within a consensus model," International Journal of Uncertainty Fuzziness and Knowledge Based Systems, vol. 16, no. 1, pp. 35-53, 2008.

[21] Y. Xu, F. J. Cabrerizo, and E. Herrera-Viedma, "A consensus model for hesitant fuzzy preference relations and its application in water allocation management," Applied Soft Computing, vol. 58, pp. 265-284, 2017.

[22] F. Shen, X. Ma, Z. Li, Z. Xu, and D. Cai, "An extended intuitionistic fuzzy TOPSIS method based on a new distance measure with an application to credit risk evaluation," Information Sciences, vol. 428, pp. 105-119, 2018.

[23] R. Ureña, F. Chiclana, G. Melançon, and E. Herrera-Viedma, "A social network based approach for consensus achievement in multiperson decision making," Information Fusion, vol. 47, pp. 72-87, 2019. 